\title{
Fluoroscopy-only Based Interventional Closure of the Left Atrial Appendage in Patients With Atrial Fibrillation
}

Henning Ebelt ( $\square$ henningebelt@gmail.com )

Catholic Hospital Erfurt https://orcid.org/0000-0003-2465-1812

\section{Luisa Linss}

Catholic Hospital Erfurt

\section{Peter Roehl}

Catholic Hospital Erfurt

Uta Pertschy

Catholic Hospital Erfurt

Andreas Schwenzky

Catholic Hospital Erfurt

\section{Research Article}

Keywords: left atrial appendage, atrial fibrillation, LAAC

Posted Date: February 21st, 2022

DOI: https://doi.org/10.21203/rs.3.rs-1305764/v1

License: (c) (1) This work is licensed under a Creative Commons Attribution 4.0 International License. Read Full License 


\section{Abstract}

Objectives: The aim of this study was to determine whether echocardiography is dispensible during interventional LAA closure when preplanning is done by cardiac CT.

Background: Interventional closure of the LAA is an established treatment alternative for patients with atrial fibrillation who are at risk for bleeding events under oral anticoagulation. Today, most LAAC procedures are guided by TEE which, however, leads to the need for patient sedation and might even cause direct harm to the patient. Technical improvements in device design and interventional experience might allow to abstain from TEE.

Methods: Fluoro-FLX is a prospective single center study to evaluate how often TEE leads to a procedural change during interventional LAAC if a dedicated CT planning algorithm is applied and the newest generation of the WATCHMAN device (WATCHMAN FLX) is used. The study hypothesis is that under these circumstances fluoroscopy-only guided LAAC is non-inferior to a TEE-guided approach, the primary endpoint is defined as the percentage of LAAC procedures without interruption by TEE. All procedures are pre-planned by cardiac CT and finally guided by fluoroscopy only while TEE is carried out in the background during the intervention for safety reasons.

Results: In none of 31 consecutive patients, TEE led to a change of the fluoroscopy-guided LAAC (success ratio: 1.00; $\mathrm{Cl}: 0.94-1.00$ ) thereby meeting the primary endpoint of non-inferiority. There were no procedure-related adverse cerebrovascular events (no pericardial effusion, TIA, stroke, systemic embolism, device embolism, death).

Conclusions: Our data suggest that it is feasible to perform LAAC under sole fluoroscopic guidance if preplanning is performed by cardiac CT and WATCHMAN FLX is used.

\section{Introduction}

Atrial fibrillation (AF) which is the most common cardiac arrhythmia in advanced age is associated with the risk of intracardiac thrombus formation and the occurrence of stroke and systemic embolism. Oral anticoagulation (OAC) is nowadays the standard therapy for patients with AF who have an increased risk of stroke (1). However, a number of these patients develop bleeding complications that require discontinuation of OAC. In this situation, interventional closure of the left atrial appendage (LAA closure, LAAC) represents an established treatment alternative (2).

In the majority of cases, left atrial appendage closure (LAAC) is performed as a transvenous catheterbased procedure. To enable correct placement of the occluder, the procedure is usually guided by both angiography (x-ray fluoroscopy) and transesophageal echocardiography (TEE). However, the use of TEE requires either general anesthesia or at least conscious sedation, which both are associated with potential threats and inconveniences to the patient. In addition, TEE per se carries the risk of injury to the pharynx and esophagus. In a recent study it has been reported that in patients who underwent a TEE- 
guided cardiac intervention, lesions in the esophagus caused by TEE could be detected in $86 \%$ of all cases by endoscopy (3). Another study showed that prolonged use of TEE during structural interventions resulted in clinically relevant complications in $6.1 \%$ of cases (4).

Against this background, it seems desirable to be able to perform LAAC without TEE. This is possible, for example, by using intracardiac echocardiography (ICE) instead. The feasibility of ICE-guided LAAC has been shown previously (5), but this method requires a second or enlarged transseptal access and is associated with an increase of procedure-related costs thereby limiting its clinical use. Alternatively, it also has been described that LAAC might be performed without any ultrasound-based imaging modality by the implementation of 3D rotational angiography (6). However, this technique is depending on a temporary "cardiac arrest" through tachypacing and leads to a rise of exposure to X-ray contrast medium and radiation, respectively.

In principle, the use of TEE during LAAC is considered helpful or necessary especially at certain key steps during the procedure. First, TEE is used to guide transseptal puncture (TSP) to determine the 'optimal' puncture site within the interatrial septum thereby potentially achieving the most favorable access to the LAA which could facilitate the further course of the procedure. Nevertheless, so far there are no clinical studies which actually have shown an advantage of TEE-guided TSP on the final result of the LAAC procedure in respect to device positioning or completeness of LAA sealing etc.. Second, the use of TEE during TSP is considered to reduce the risk of pericardial tamponade. However, TSP is nowadays performed in daily routine without TEE in a large number of other cardiac interventions (e.g. pulmonary vein isolation etc.) without a relevant risk of cardiac tamponade so that TEE can be considered dispensable in this regard. Third, TEE is used to rule out intracardiac thrombi and to determine the size of the LAA in order to select the suitable size of the LAAC device although these tasks can be completed already before the implantation procedure either by diagnostic TEE or preferably by cardiac CT. Finally, the perhaps most important role of TEE in LAAC is to confirm the correct positioning of the occluder before the device is released definitely. For the WATCHMAN, so called "PASS criteria" are applied (position, anchor, size, seal) which according to IFU should be checked by TEE albeit in principle it is possible to perform this evaluation also based on fluoroscopy.

Against the background of these explanations, the assumption is justified that nowadays LAAC can be performed safely under sole fluoroscopic guidance without the need for intraprocedural ultrasound. This hypothesis is based on the existing clinical experience regarding the technique of left atrial appendage closure and the technical improvements that have been implemented into the development of new generation LAAC systems.

\section{Methods}

Fluoro-FLX is a prospective, single center trial performed at Catholic Hospital "St. Johann Nepomuk" Erfurt, Germany; ideas regarding study design were adopted from (6). The trial was approved by the ethics committee of the medical association of Thuringia and is registered at German Registry of Clinical 
Trials, DRKS00023464. Consecutive patients that were planned for LAAC were screened for study inclusion. Inclusion criteria were age > 18 years, atrial fibrillation (paroxysmal, persistent, or permanent) with planned interventional left atrial appendage closure using the WATCHMAN FLX, signed informed consent, exclusion of intracardiac thrombi by cardiac CT within 72 hours before LAAC, and no long-term interruption of therapeutic anticoagulation before the start of LAAC (i.e. last administration of NOAC or LMW heparin within 48 hours before LAAC). Exclusion criteria were history of ASD/ PFO closure, contraindication regarding TEE, lack of informed consent, and anticipated inability to perform 3 months follow-up (including TEE).

After obtaining informed consent, CT images (64 slice, Somatom Perspective, Siemens Healthcare) were analyzed using 3D LEONARDO Workstation (Siemens Healthcare) i) to exclude intracardiac thrombi, ii) to predict two optimal C-arm angulations with an orthogonal projection of the LAA ostium with a distance of at least $60^{\circ}$, iii) to measure the diameter of the LAA landing zone. During LAAC, all patients were sedated by continuous i.v. administration of propofol $1 \%$ with the addition of fentanyl as required. The TEE probe was inserted at the beginning of the procedure, the monitor of the ultrasound device was arranged in a way that it could only be seen by the echo physician but not by the interventionalist. After puncturing the right femoral vein, a standardized transseptal puncture (TSP) was prepared with fluoroscopic guidance. When the transseptal needle was in place, confirmation of a safe puncture site was confirmed by the echo physician (checkpoint 1) before TSP was done. Anticoagulation was ensured by i.v. administration of unfractionated heparin with a target ACT of 250-300 seconds. After TSP, an Amplatz Super Stiff wire was placed in a left pulmonary vein and the transseptal TruSeal sheath was advanced into the left atrium (LA). A pigtail catheter was then used to obtain angiography of the LAA at least in one of the two predefined angiographic projections. The dimensions of the LAA were determined by the interventionalist and compared with the measurements that had been obtained by CT. Afterwards, the selection of the appropriate size of the WM FLX was made by the interventionalist and documented and communicated to the echo physician. The echo physician had to confirm correct device sizing (checkpoint 2), then the device packaging was opened and the WM FLX was prepared and stepwise placed into the LAA according to standard techniques. Adequate device compression and the proper position in the LAA ostium as well as the absence of a para-device leak were determined at the 2 angiographic projections that had been selected previously on the basis of the CT preplanning. If according to the opinion of the interventionalist all release criteria were met, the echo physician had to confirm the correct device position (checkpoint 3) and the occluder was then released from the catheter. At any time during the procedure, the echo physician could interrupt the procedure if complications or safety-relevant abnormalities were occurring. Any disclosure of the TEE to the interventionalist was recorded in the study documentation. Follow-up TEE was carried out 3 months after LAAC.

\section{Study end point}

The primary end point of the study was defined as the percentage of LAAC procedures without interruption by TEE. For statistical analysis, a non-inferiority testing was used with a presumed efficacy of 
$97.5 \%$ and a margin of equivalence of $8 \%$ leading to a sample size of 30 patients at a power of $ß=0.80$.

\section{Results}

Between 28.10.2020 and 20.05.2021, 34 consecutive patients were screened for study inclusion (fig.1). All CT datasets were suitable to assess the anatomy of the LAA and to perform LAAC pre-planning. In 2 cases thrombi could not be definitely ruled out by CT due to poor contrast penetration into the LAA so that a pre-implantation TEE using echo contrast agent (SonoVue) was done additionally as descibed before (7). In 31 patients, LAAC was attempted and in 30 patients LAAC could be technically completed. In one patient, no adaquate positioning of the WM FLX device could be achieved (reversed chicken wing anatomy). In that case, a conventional approach using standard TEE-guiding was undertaken directly after the study procedure which also did not lead to technical success.

Demographic and clinical data are given in tab. 1, procedural characteristics of the LAA closure and implantation results after the release of the WM FLX as well as at the 3 months follow-up are given in tab. 2. In none of the cases, TEE led to an interruption or change of the implantation procedure thereby reaching the primary study end point (fig. 2).

During the course of the study, it became evident that the renunciation of TEE led to a slight increase in contrast medium in comparison to previous experiences from our center (8). Therefore, after 20 patients we started to apply biplanar fluoroscopy which led to a reduction of contrast exposure as expected (monoplane: $77 \pm 26 \mathrm{ml}$; biplane: $55 \pm 21 \mathrm{ml} ; \mathrm{p}=0.02$ ) with no significant change in radiation dose (monoplane: $19 \pm 11 \mathrm{~Gy}^{*} \mathrm{~cm}^{2}$; biplane: $24 \pm 20 \mathrm{~Gy}^{*} \mathrm{~cm}^{2} ; \mathrm{p}=0.45$ ) or procedure time (monoplane: $33 \pm 7$ min; biplane: $30 \pm 10 \mathrm{~min} ; \mathrm{p}=0.38$ ), respectively.

As seen from tab. 3, no adverse cerebrovascular clinical endpoints were observed during the study. However, in one patient an adverse event related to the TEE was documented (pharyngeal bleeding).

\section{Discussion}

LAAC is nowadays a proven alternative for patients with atrial fibrillation and a high thromboembolic risk who are not considered suitable for oral anticoagulation $(9,10)$. Since the early days of this technology, a number of improvements have been implemented into new devices and interventional strategies including imaging so that the procedure of LAAC has become both more effective and safer $(9,11)$. Today, the vast majority of cases are still performed under guidance of TEE although this contains several draws backs deriving from the need of patient sedation and the semi-invasive nature of the TEE $(3,4)$. Our study presented here shows that it is feasible to restrain from TEE if the newest generation of the LAA closure device WATCHMAN FLX is used and if a dedicated CT-based planning of the procedure is done.

So far, there's only limited evidence regarding the question to which extent TEE guidance indeed has an influence on the procedure of LAAC and whether echo guidance might perhaps be dispensable $(12,13)$. 
Recently, a paper was published describing the experience of a single high volume center where 811 LAAC procedures were done either with $(\mathrm{N}=262)$ or without TEE guidance $(\mathrm{N}=549)$ between 2009 and 2020 (14). In this retrospective analysis, the echo-guided approach was linked to favorable efficacy and safety outcomes. However, there was obviously no CT-based procedure planning and in the group with sole fluoroscopic guidance WATCHMAN FLX was not used at all and so that these finding should not be compared directly to our data.

In our study, we have implemented a dedicated CT-planning algorithm in order to increase accuracy and success rate of a fluoroscopy-only based LAAC. To achieve an optimal implantation result and especially to avoid device embolization, the WM FLX should be positioned at or just distal to the LAA ostium. This might be difficult to evaluate by fluoroscopy if the angulation of the $\mathrm{C}$-arm is not aligned perpendicular to the LAA ostial plane. In our study, we have overcome this drawback using cardiac CT to predict patientspecific optimal $\mathrm{C}$-arm angulations that were used during the intervention. Additionally, it is important to ensure that all lobes of the LAA are finally covered and thereby sealed by the LAA closure device so that there is no relevant residual paradevice leakage $(\leq 5 \mathrm{~mm} ;(15))$. Assuming that a paradevice contrast leak is safely identified by fluoroscopy if the contrast medium exceeds the metal border of the WM FLX by at least $1.5 \mathrm{~mm}$, all leaks larger than $3 \mathrm{~mm}$ can be the identified if two angiographic views are checked with a distance in angulation of at least $60^{\circ}$ (e.g. RAO 30 / LAO 30). Again, the CT-based prediction of the patient-specific optimal $\mathrm{C}$-arm angulations allow this leak detection with high accuracy.

\section{Study limitations}

Our study consists only of 31 LAAC cases which limits the statistical strength especially regarding clinical outcomes. Additionally, 3 months follow-up TEE was available only in 23 patients which was mainly caused by the ongoing COVID-19 pandemic. According to the study protocol, only procedures with the WM FLX were included into the trial so that no comparison between different LAAC devices can be given.

\section{Conclusions}

Our prospective single center study shows that the diagnostic yield of TEE during LAAC is rather limited so that TEE might be considered dispensible if the newest generation of the LAA closure device WATCHMAN FLX is used and if a dedicated CT-based pre-planning of the procedure is done.

\section{Abbreviations}




\begin{tabular}{|ll|}
\hline LAA & left atrial appendage \\
\hline LAAC & left atrial appendage closure \\
\hline AF & atrial fibrillation \\
\hline CT & computed tomography \\
\hline TEE & transesophageal echocardiography \\
\hline OAC & oral anticoagulation \\
\hline NOAC & non vitamin $K$ antagonist oral anticoagulants \\
\hline TSP & transseptal puncture \\
\hline WM & WATCHMAN \\
\hline
\end{tabular}

\section{Declarations}

Funding: N.A:

Competing Interests: HE has received consulting fees/ honoraria for lectures from Pfizer, Boehringer Ingelheim, and Boston Scientific. HE has received research grants from Boston Scientific and Siemens Healthcare. The other authors have nothing to disclose.

Author Contributions: All authors contributed to the study conception and design. Material preparation, data collection and analysis were performed by HE, LL, AS, UP, and PR. The first draft of the manuscript was written by $\mathrm{HE}$ and all authors commented on previous versions of the manuscript. All authors read and approved the final manuscript.

Ethics approval: The trial was approved by the ethics committee of the medical association of Thuringia and is registered at German Registry of Clinical Trials, DRKS00023464.

Consent to participate: Informed consent was obtained from all individual participants included in the study.

Consent to publish: N.A.

Availability of data and material: N.A.

\section{References}

1. Kirchhof P, Benussi S, Kotecha D et al. 2016 ESC Guidelines for the Management of Atrial Fibrillation Developed in Collaboration With EACTS. Rev Esp Cardiol (Engl Ed) 2017;70:50.

2. Holmes DR, Jr., Reddy VY. Left Atrial Appendage and Closure: Who, When, and How. Circ Cardiovasc Interv 2016;9:e002942. 
3. Freitas-Ferraz AB, Bernier M, Vaillancourt R et al. Safety of Transesophageal Echocardiography to Guide Structural Cardiac Interventions. J Am Coll Cardiol 2020;75:3164-3173.

4. Freitas-Ferraz AB, Rodes-Cabau J, Junquera Vega $L$ et al. Transesophageal echocardiography complications associated with interventional cardiology procedures. Am Heart J 2020;221:19-28.

5. Ribeiro JM, Teixeira R, Puga L, Costa M, Goncalves L. Comparison of intracardiac and transoesophageal echocardiography for guidance of percutaneous left atrial appendage occlusion: A meta-analysis. Echocardiography 2019;36:1330-1337.

6. De Potter T, Chatzikyriakou S, Silva E, Van Camp G, Penicka M. A Pilot Study for Left Atrial Appendage Occlusion Guided by 3-Dimensional Rotational Angiography Alone. JACC Cardiovasc Interv 2018;11:223-224.

7. Ebelt $\mathrm{H}$, Offhaus $\mathrm{A}$, Wiora $\mathrm{M}$ et al. Impact of ultrasound contrast agent on the detection of thrombi during transoesophageal echocardiography. Open Heart 2019;6:e001024.

8. Ebelt $\mathrm{H}$, Domagala $\mathrm{T}$, Offhaus $\mathrm{A}$ et al. Fusion Imaging of X-ray and Transesophageal Echocardiography Improves the Procedure of Left Atrial Appendage Closure. Cardiovasc Drugs Ther 2020;34:781-787.

9. Kar S, Doshi SK, Sadhu A et al. Primary Outcome Evaluation of a Next-Generation Left Atrial Appendage Closure Device: Results From the PINNACLE FLX Trial. Circulation 2021;143:1754-1762.

10. Hildick-Smith D, Landmesser U, Camm AJ et al. Left atrial appendage occlusion with the Amplatzer Amulet device: full results of the prospective global observational study. Eur Heart J 2020;41:28942901.

11. Freeman JV, Varosy P, Price MJ et al. The NCDR Left Atrial Appendage Occlusion Registry. J Am Coll Cardiol 2020;75:1503-1518.

12. Zhang $X$, Jin $Q$, Kong $D$ et al. Comparison of fluoroscopy and transesophageal echocardiogram for intra-procedure device surveillance assessment during implantation of Watchman. Int $\mathrm{J}$ Cardiol 2021;324:72-77.

13. Yuniadi Y, Hanafy DA, Raharjo SB, Yugo D. Left atrial appendage closure device implantation guided with fluoroscopy only: Long-term results. J Arrhythm 2019;35:262-266.

14. Galea R, Raber L, Fuerholz M et al. Impact of Echocardiographic Guidance on Safety and Efficacy of Left Atrial Appendage Closure: An Observational Study. JACC Cardiovasc Interv 2021;14:1815-1826.

15. Viles-Gonzalez JF, Kar S, Douglas $P$ et al. The clinical impact of incomplete left atrial appendage closure with the Watchman Device in patients with atrial fibrillation: a PROTECT AF (Percutaneous Closure of the Left Atrial Appendage Versus Warfarin Therapy for Prevention of Stroke in Patients With Atrial Fibrillation) substudy. J Am Coll Cardiol 2012;59:923-9.

\section{Tables}

Tab. 1: Baseline demographic, clinical charecteristics and CT-derived parameters of patients undergoing LAA closure $(\mathrm{N}=31)$. 


\begin{tabular}{|ll|}
\hline parameter & value (\%) \\
\hline demographics & \\
\hline female sex & $13(45 \%)$ \\
\hline AF pattern & $10(33 \%)$ \\
\hline paroxysmal & $4(13 \%)$ \\
\hline persistent & $16(53 \%)$ \\
\hline permanent & $28(93 \%)$ \\
\hline hypertension & $15(50 \%)$ \\
\hline diabetes & $8(27 \%)$ \\
\hline history of stroke & $9(30 \%)$ \\
\hline coronary artery disease & $78.9 \pm 7.3$ \\
\hline age (years) & $171 \pm 11$ \\
\hline height (cm) & $84 \pm 20$ \\
\hline weight (kg) & $4.6 \pm 1.4$ \\
\hline CHA2DS2VASc & $3.2 \pm 1.0$ \\
\hline HASBLED & $52 \pm 21$ \\
\hline eGFR (ml/min*1.73m2) & value \\
\hline baseline CT & $25.4 \pm 3.5$ \\
\hline LAA size (mm) & $35.6 \pm 10.5 /-30.5 \pm 9.4$ \\
\hline predicted C-arm angulation & \\
\hline first projection & $-29.0 \pm 8.7 / 0.3 \pm 14.2$ \\
\hline second projection & \\
\hline
\end{tabular}

Tab. 2: Procedural characteristics of LAA closure and implantation results. 


\begin{tabular}{|c|c|}
\hline parameter & value \\
\hline technical implant success & $30 / 31(96.8 \%)$ \\
\hline procedure time & $32 \pm 8 \mathrm{~min}$ \\
\hline \multicolumn{2}{|l|}{ time from venous access to } \\
\hline TSP & $7 \pm 5 \min$ \\
\hline LAA angiography & $17 \pm 7 \mathrm{~min}$ \\
\hline opening of device package & $19 \pm 7 \min$ \\
\hline final placement of device according to fluoroscopy & $29 \pm 8 \mathrm{~min}$ \\
\hline release of device & $30 \pm 8 \min$ \\
\hline access site closure & $32 \pm 8 \mathrm{~min}$ \\
\hline size of LAA in angiography (mm) & $24.0 \pm 3.3$ \\
\hline contrast medium (ml) & $70 \pm 26$ \\
\hline radiation $\left(\mathrm{Gy} * \mathrm{~cm}^{2}\right)$ & $20.8 \pm 14.2$ \\
\hline number of devices used & $1.0 \pm 0.0$ \\
\hline \multicolumn{2}{|l|}{ size of implanted device $(\mathrm{mm})$} \\
\hline 20 & $0(0 \%)$ \\
\hline 24 & $6(20 \%)$ \\
\hline 27 & $8(27 \%)$ \\
\hline 31 & $12(40 \%)$ \\
\hline 35 & $4(13 \%)$ \\
\hline \multicolumn{2}{|l|}{ TEE measurements after device release $(\mathrm{N}=30)$} \\
\hline \multicolumn{2}{|l|}{ compression (\%) } \\
\hline minimum & $13.0 \pm 5.8$ \\
\hline maximum & $20.7 \pm 5.9$ \\
\hline mean & $16.9 \pm 5.5$ \\
\hline \multicolumn{2}{|l|}{ maximum leak } \\
\hline no & $29(97 \%)$ \\
\hline$<3 \mathrm{~mm}$ & $1(3 \%)$ \\
\hline 3 to $5 \mathrm{~mm}$ & $0(0 \%)$ \\
\hline
\end{tabular}




\begin{tabular}{|c|c|}
\hline$>5 \mathrm{~mm}$ & $0(0 \%)$ \\
\hline \multicolumn{2}{|l|}{ max. protrusion towards LA (mm) } \\
\hline minimum & $2.0 \pm 3.0$ \\
\hline maximum & $9.7 \pm 3.7$ \\
\hline mean & $6.0 \pm 3.1$ \\
\hline interruption or change of interventional procedure due to TEE finding & $0(0 \%)$ \\
\hline adverse event due to TEE & $1(3 \%)$ \\
\hline \multicolumn{2}{|l|}{ TEE measurements after 3 months $(\mathrm{N}=23)$} \\
\hline \multicolumn{2}{|l|}{ compression (\%) } \\
\hline minimum & $11.5 \pm 6.9$ \\
\hline maximum & $20.5 \pm 7.5$ \\
\hline mean & $16.0 \pm 6.4$ \\
\hline \multicolumn{2}{|l|}{ maximum leak } \\
\hline no & 20 (87\%) \\
\hline$<3 \mathrm{~mm}$ & $2(9 \%)$ \\
\hline 3 to $5 \mathrm{~mm}$ & $1(4 \%)$ \\
\hline$>5 \mathrm{~mm}$ & $0(0 \%)$ \\
\hline \multicolumn{2}{|l|}{ maximal protrusion towards LA (mm) } \\
\hline minimum & $2.4 \pm 3.7$ \\
\hline maximum & $8.9 \pm 4.1$ \\
\hline mean & $5.7 \pm 3.7$ \\
\hline
\end{tabular}

Tab. 3: Clinical endpoints after LAAC until hospital discharge and given medication. 


\begin{tabular}{|ll|}
\hline clinical endpoint & value \\
\hline pericardial efflusion & $0(0 \%)$ \\
\hline TIA & $0(0 \%)$ \\
\hline stroke & $0(0 \%)$ \\
\hline systemic embolism & $0(0 \%)$ \\
\hline device embolism & $0(0 \%)$ \\
\hline death & $0(0 \%)$ \\
\hline medication & \\
\hline aspirin & $29(97 \%)$ \\
\hline clopidogrel & $28(93 \%)$ \\
\hline ticagrelor & $0(0 \%)$ \\
\hline prasugrel & $0(0 \%)$ \\
\hline DAPT & $27(90 \%)$ \\
\hline VKA & $0(0 \%)$ \\
\hline NOAC & $2(7 \%)$ \\
\hline NOAC + antiplatelet & $2(10 \%)$ \\
\hline
\end{tabular}

Figures 
Screening: 34 consecutive patients

3 screen failures:

2 pat. with thrombus in LAA

1 pat. without CT

31 patients with attempted LAAC

1 pat. with unsuitable anatomy

Figure 1

Patient flow.

\section{Percentage of procedures not changed by TEE}

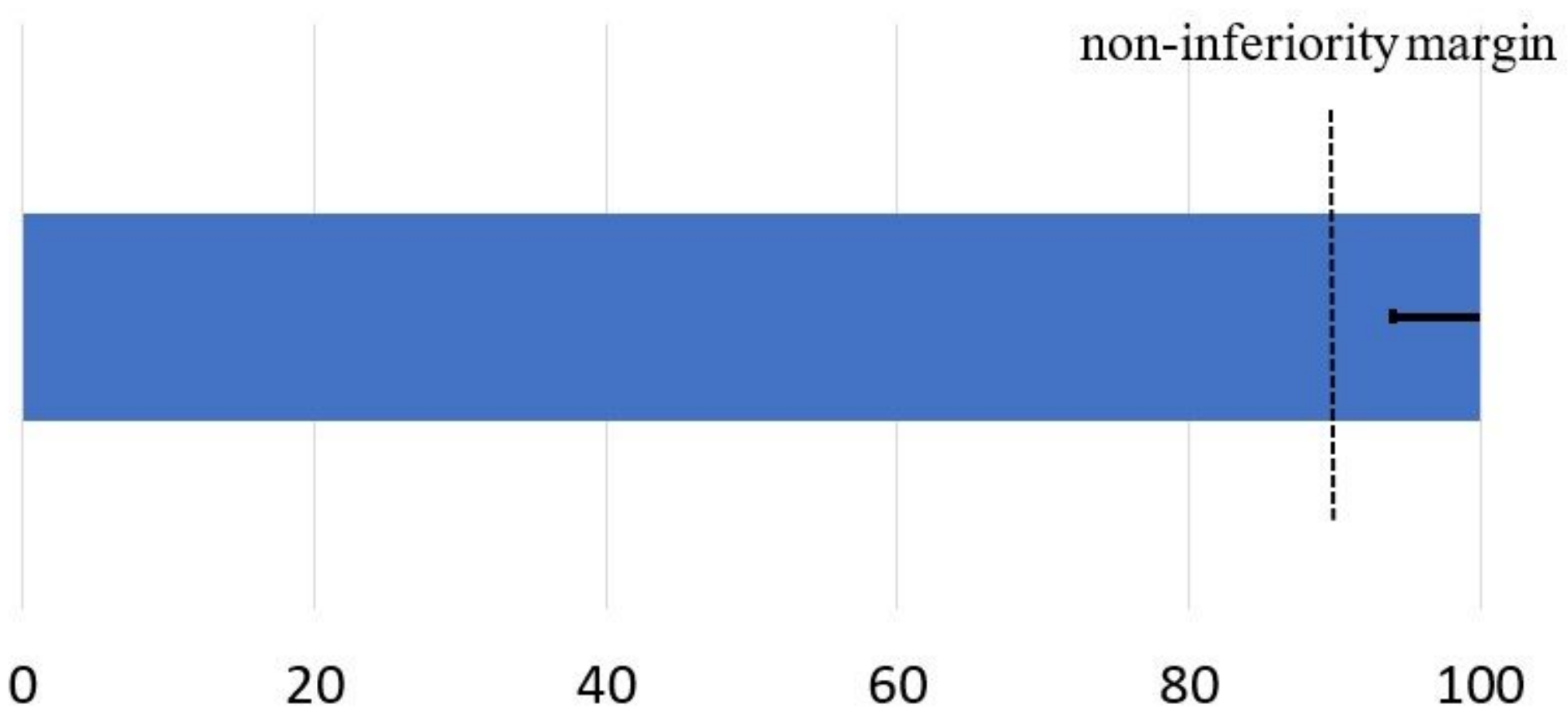


Figure 2

Percentage of LAAC procedures that have not been influenced by TEE findings ( $\mathrm{N}=31$ ). The error bar shows the $95 \%$ confidence interval (Likelihood); it does not cross the specified non-inferiority margin so that the primary endpoint is met. 